\title{
Applications of Field-Reversal and Angle-Dependent XMCD Techniques to Mn-Based Diluted Magnetic Materials
}

\author{
Zhiying GUO*, Juncai DONG, Yidong ZHAO, Dongliang CHEN \\ Institute of High Energy Physics, Chinese Academy of Sciences, Beijing 100049, China \\ cross ref http://dx.doi.org/10.5755/j01.ms.25.2.19135
}

Received 27 September 2017; accepted 30 March 2018

\begin{abstract}
Recent progress of the soft X-ray magnetic circular dichroism (XMCD) techniques and relevant applications at beamline 4B7B in Beijing Synchrotron Radiation Facility are reported here. The key progress of the XMCD techniques include i) improvements in the accuracy and sensitivity of XMCD measurements by fast-reversing magnetic field with electromagnet, and ii) establishment an angle-dependent experimental method for obtaining the magnetic anisotropy information. These techniques have been applied to investigate the interface ferromagnetism and magnetic anisotropy of two Mn-based materials, i.e. Fe/(Ga, Mn)As and $\mathrm{La}_{2 / 3} \mathrm{Sr}_{1 / 3} \mathrm{MnO}_{3} / \mathrm{SrTiO}_{3}$ bilayer heterostructures. An enhanced XMCD signal has been observed at the Fe $L_{2,3}$-edges whereas a relative small but unambiguous Mn XMCD signal with opposite sign has been detected which indicates an antiferromagnetic coupling at $\mathrm{Fe} /(\mathrm{Ga}, \mathrm{Mn})$ As interfaces. A comparative study of the stoichiometric and nonstoichiometric $\mathrm{La}_{2 / 3} \mathrm{Sr}_{1 / 3} \mathrm{MnO}_{3} / \mathrm{SrTiO}_{3}$ bilayers clearly demonstrates that the oxygen vacancies degrade the magnetic properties of the perovskite manganese oxide film. These achievements benefit from the established field-reversal and angle-dependent XMCD techniques, which will make it possible to extend the research field of our devices from ferromagnetic to paramagnetic or diluted magnetic semiconductor system.

Keywords: X-ray magnetic circular dichroism, synchrotron radiation, electromagnet, magnetic materials.
\end{abstract}

\section{INTRODUCTION}

X-ray magnetic circular dichroism (XMCD) has been shown to be a powerful tool to measure the elementspecific magnetic moments [1]. After the proposal of the XMCD sum rules [2,3], the spin and orbital moments can be independently determined, which make the XMCD into a quantitative magnetometry tool. This technique is now widely used to study the magnetic properties of various ferromagnetic (FM) and ferrimagnetic systems $[4,5]$. Furthermore, benefit from the high field electromagnet [6] and superconducting magnets [7], even very small magnetic moments can be obtained from paramagnetic materials or diluted magnetic semiconductors (DMSs) [8].

A new soft X-ray XMCD setup has been designed and constructed in our recent work [9] at the 4B7B beamline, which is a bending magnet beamline at Beijing Synchrotron Radiation Facility (BSRF), equipped with a varied-line-spacing plane grating monochromator. Demonstration XMCD experiments in ferromagnetic 3d transition metals $(\mathrm{Fe}, \mathrm{Co}$ and $\mathrm{Ni})$ films and ultrathin films were performed. In order to extend the research field to Mn-based diluted magnetic materials, especially unravel the microscopic origin of magnetism in Mn doped DMSs and perovskite manganese oxide films, the following two techniques have been developed for our XMCD setup:

(i) Fast-reversing magnetic field techniques. A variety of switching and modulation techniques (such as currentreversal, mechanically reversal the superconducting coils, fast helicity reversal by kicker system or phase retarder, moving the magnet array of undulator, high-speed chopper, etc.) have been reported with the development of advanced

\footnotetext{
${ }^{*}$ Corresponding author. Tel.: +86-10-88235156; fax: +86-10-88235156 E-mail address: zyguo@ihep.ac.cn (Z. Guo)
}

insertion devices and third-generation light source [10]. These modulation techniques can be divided into two kinds of equivalent categories: the magnetic-field switching method and the photon-helicity switching method. Moreover, the helicity switching technique combined with the lock-in amplifier has been used to improve the accuracy of the XMCD measurement [11]. Nevertheless, the helicity switching method cannot be achieved for a bending magnet beamline, and current reversal is more reliable and convenient compared with mechanically reversal the coils. For these reasons, the current-reversal method has been established for our system.

(ii) Angle-dependent XMCD method for magnetic anisotropy. The orbit magnetic moment is directly linked to the dichroism intensity, while the determination of the spin moment is more complicated by the presence of a correction magnetic dipole term in the spin sum rule [12]. To overcome this difficulty, Stöhr and König [13] proposed angle-dependent measurements of XMCD, which cancel the magnetic dipole term. By use of this angledependent method, the anisotropy of the orbital magnetic moment can also be determined, and it is directly related to the magnetocrystalline anisotropy. After that, various polar angle and azimuthal angle dependent XMCD has been widely used to study the magnetic anisotropy [14-18]. In addition to the longitudinal angle-dependent XMCD (magnetic field B is parallel to the photon helicity h.), Dürr and van der Laan discussed angle-dependent XMCD spectra in the transverse arrangements $(B \perp h)$ [19-21]. Mamiya [22] studied the magnetic states of a Fe layer on $\mathrm{MgO}$ tunnel barrier by angle-dependent XMCD in the longitudinal and transverse geometry.

The above-mentioned field-reversal and angledependent techniques would be very useful to extract the weak magnetic signals at the interface and the magnetic 
anisotropy information. Thus, this paper aims to discuss the establishment of these experimental methods and possible applications for the Mn-based diluted magnetic materials.

\section{EXPERIMENTAL METHODS AND TECHNIQUES}

\subsection{The magnetic field or helicity switching techniques}

This method was accomplished by a high precision digital power supply, by which the magnet field can be reversed by revering the direction of the current in the coils. The power supply (180A, 20V) was customized from Beijing Boxing Keyuan technology Co., Ltd. In order to reduce the fluctuation of the magnetic field strength, the current stability is better than $10^{-3}$ and the total harmonic distortion is lower than $0.5 \%$. The ramping rate is suitable for point-by-point field reversal method, and the reversal frequency of the current was up to $2 \mathrm{~Hz}$. A demonstration experiment of each point field reversal method and the Labview control interface of the power supply are shown in the Figure S1 (Supplementary Material). The differential XMCD signal can be achieved directly, thus effectively eliminating the artificial error and the influence of slow drifts of the photon source.

\subsection{The angle-dependent XMCD method for magnetic anisotropy}

In the Bruno model, the difference between the orbital moments along the easy and hard directions is proportional to the magnetocrystalline anisotropy. For samples with uniaxial anisotropy, one obtains $\Delta E_{s o}=\zeta / 4 \mu_{B}\left(m_{\text {orb }}^{\perp}-m_{\text {orb }}^{\square}\right)$ [13], where $\zeta$ is the spin-orbit constant, $\mu_{B}$ is the Bohr magneton, the $m_{\text {orb }}^{\perp}$ and $m_{\text {orb }}^{\square}$ are the orbital magnetic moment along the film normal and in the plane. For an experimental geometry where magnetic field $H_{e x t}$ are at an angle $\gamma$ with respect to the surface normal, the orbital moment $m_{o}^{\gamma}$ along the field direction can be directly determined by use of the sum rule. If $H_{e x t}$ is sufficiently large to magnetically saturate the sample, then $m_{\text {orb }}^{\gamma}=m_{\text {orb }}^{\square} \sin ^{2} \gamma+m_{\text {orb }}^{\perp} \cos ^{2} \gamma$, such that measurements at two angles are needed to determine $m_{o r b}^{\perp}-m_{o r b}^{\square}$. Considering absorption measurements are difficult at grazing incidence where $\gamma>80^{\circ}$, we can obtain the accurate value of the inplane orbital magnetic moment $\left(m_{\text {orb }}^{\square}\right)$ by multi-angular measurements.

In order to verify the above mentioned ideas and show potential applications for the two techniques/methods, we study the magnetic properties and magnetic anisotropy of two typical Mn-based magnetic materials, Fe/(Ga,Mn)As and $\mathrm{La}_{2 / 3} \mathrm{Sr}_{1 / 3} \mathrm{MnO}_{3} / \mathrm{SrTiO}_{3}$ heterostructures.

\section{APPLICATION}

\subsection{Application to FM/DMSs bilayer}

( $\mathrm{Ga}, \mathrm{Mn}) \mathrm{As}$ has been considered as one of the most promising spintronics materials due to its high spin polarizaiton and ferromagnetism induced by doped hole carriers. However, the main obstacle for the practical application of $(\mathrm{Ga}, \mathrm{Mn}) \mathrm{As}$ is their low Curie temperatures. Remarkably, room-temperature ferromagnetism of the Mn atoms was reported at the interface of $\mathrm{Fe} /(\mathrm{Ga}, \mathrm{Mn}) \mathrm{As}$ heterostructures due to the magnetic proximity effect of thin Fe overlayer [23]. Furthermore, there are some contrary report about the ferro- or antiferromagnetic coupling between the $\mathrm{Mn}$ ions and the Fe layer [24-27]. Recently, proximity effect induced enhancement of the Curie temperature and spin ordering has also been reported $[28,29]$. Those studies were mainly focused on the interfacial proximity effect as well as the possibility of spin injection from the FM layer to the DMS layer. On the other hand, the magnetic anisotropy of FM/DMS structures has not been extensively investigated yet [30], especially not investigated by the angle-dependent X-ray magnetic circular dichroism (XMCD) method, although the anisotropy of the GaMnAs films has been widely studied. So, we can believe that the magnetic anisotropy research about the $\mathrm{Fe} /(\mathrm{Ga}, \mathrm{Mn})$ As bilayer may exhibit other interesting results and it is helpful for understanding the mechanism of the proximity induced magnetic ordering and spin ordering.

The $\mathrm{Fe} /(\mathrm{Ga}, \mathrm{Mn})$ As bilayer used in this study were grown by molecular beam epitaxy (MBE) on semiinsulating GaAs substrates. The structures of the sample is $\mathrm{Al}(1.6 \mathrm{~nm}) / \mathrm{Fe}(2 \mathrm{~nm}) / \mathrm{Ga}_{0.95} \mathrm{Mn}_{0.05} \mathrm{As}(20 \mathrm{~nm}) / \mathrm{GaAs}(001)$.

In order to determine the origin of room temperature ferromagnetism and further study the interfacial magnetic coupling in the bilayer, we performed XMCD experiments at the Fe- $L_{2,3}$ and Mn- $L_{2,3}$ absorption edges in total electron yield mode, which are summarized in Fig. 1. The XMCD experimental apparatus and the data processing have been described in Ref. 9. Fig. 1 a shows the Fe- $L_{2,3}$ XMCD spectrum of the $\mathrm{Fe} /(\mathrm{Ga}, \mathrm{Mn}) \mathrm{As}$ bilayer at an incident angle of $60 \mathrm{deg}$ with a magnetic field up to $0.4 \mathrm{~T}$ at room temperature. Fig. $1 \mathrm{~b}$ show the Mn- $L_{2,3}$ XMCD spectrum at normal incidence. All the spectra were normalized by the intensity of the incident beam and were not corrected for the incomplete circular polarization and incomplete magnetization. Large XMCD signals have been found for $\mathrm{Fe}$ elements from the overlayer, a relative small but unambiguous magnetic signal was obtained in the $\mathrm{Mn}$ spectrum of the bilayer even at room temperature. The low signal-to-noise ratio in Fig. $1 \mathrm{~b}$ is because of the limited escape depth of electrons and the low concentration (about $5 \%$ percent) of $\mathrm{Mn}$. Considering the paramagnetic phase of the bulk $(\mathrm{Ga}, \mathrm{Mn}) \mathrm{As}$ layer at $300 \mathrm{~K}$, we therefore suggest that the magnetic order of Mn may be induced by the Fe layer due to the magnetic proximity effect.

We also performed angle-dependent XMCD experiments to study the magnetic anisotropy in $\mathrm{Fe} /(\mathrm{Ga}, \mathrm{Mn}) \mathrm{As}$ bilayer. A differentially-pumped rotary platform was mounted on the XMCD chamber to provide 360 degree rotation with an angular resolution less than 1 degree. The sample holder made of oxygen-free copper is fixed at the bottom of a non-magnetic titanium alloy rod. A molybdenum sample plate $(15 \times 15 \mathrm{~mm})$ can be inserted into the sample holder. Fig. $1 \mathrm{c}$ shows a sketch of the $\mathrm{XMCD}$ experimental setup in longitudinal configurations. 

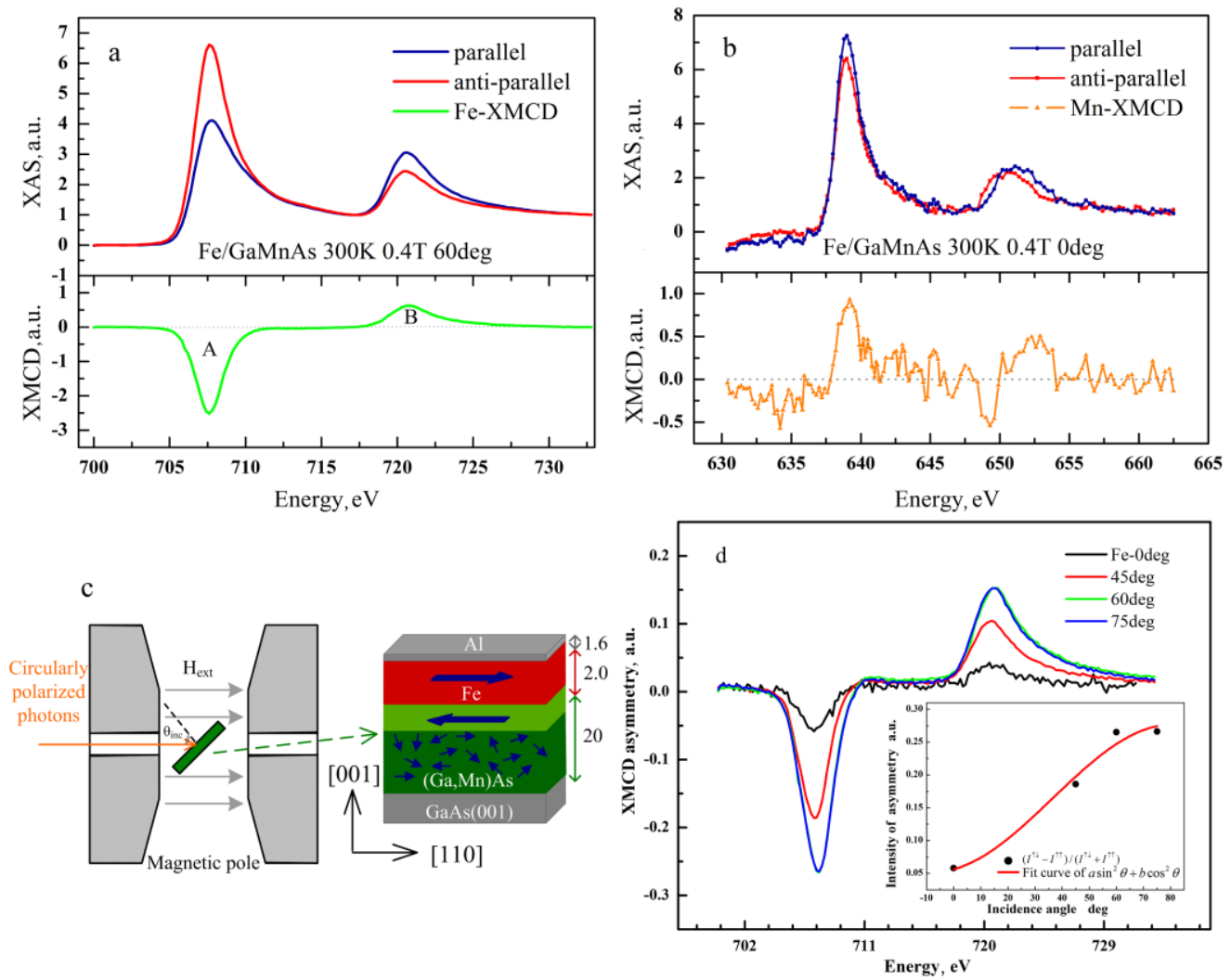

Fig. 1. XMCD spectra of $\mathrm{Fe}$ and Mn elements measured in total electron yield at room temperature and the dependence of the XMCD asymmetry on the angle of incidence: a-Fe- $L_{2,3}$ XMCD spectrum at an incident angle of $60 \mathrm{deg}$ with a magnetic field of about $0.4 \mathrm{~T}$ at room temperature; $\mathrm{b}-\mathrm{Mn}-L_{2,3} \mathrm{XMCD}$ spectrum at normal incidence at room temperature; $\mathrm{c}-$ schematic drawing of the $\mathrm{XMCD}$ experimental setup in longitudinal configurations; $d$-the XMCD spectra of Fe elements at varying angles. The inset shows the dependence of the XMCD asymmetry on the incidence angle, the data points represent the normalized XMCD intensity measured at the Fe- $L_{3}$ edge while the solid red line depicts the best-fit of the function $\mathrm{y}=a \sin ^{2} \gamma+b \cos ^{2} \gamma$

We observe enhancements of XMCD effect of Fe atoms in grazing incidence geometry as shown in Fig. $1 \mathrm{~d}$. The normalized XMCD signal is larger for the grazing angle measurements which indicates the easy magnetization axis of the $\mathrm{Fe}$ film in $\mathrm{Fe} /(\mathrm{Ga}, \mathrm{Mn}) \mathrm{As}$ is inplane. The inset of Fig. $1 \mathrm{~d}$ shows the incidence angle dependence of XMCD asymmetry, defined as $\left(I^{\uparrow \downarrow}-l^{\uparrow}\right) /\left(I^{\uparrow \downarrow}+l^{\uparrow \uparrow}\right)$, where $l^{\uparrow \downarrow}\left(I^{\uparrow \uparrow}\right)$ is the absorption for antiparallel (parallel) orientations of the sample magnetization and the incident photon spin. Notably, the experimental results of the angle dependence of XMCD asymmetry obey the above mentioned theory formula very well. By using the sum rule to the data set, we can obtain the spin and orbit moments at 0,45 and 60 degree, and then fit the orbit moments by the equation

$m_{\text {orb }}^{\gamma}=m_{\text {orb }}^{\square} \sin ^{2} \gamma+m_{\text {orb }}^{\perp} \cos ^{2} \gamma$, we obtain $m_{\text {orb }}^{\perp} \approx 0.11 \mu_{B}$, $m_{\text {orb }}^{\square} \approx 0.61 \mu_{B}$ for the Fe element in the bilayer. Compared it to our previous results in reference [9], $m_{\text {orb }}^{\perp}$ is slightly larger than the orbit moments of bulk Fe, and the $m_{o r b}^{\square}$ is 7.6 times larger which indicate the presence of a huge magnetic anisotropy in the bilayer.

\subsection{Application to perovskite manganese oxide}

$\mathrm{La}_{1-\mathrm{x}} \mathrm{Sr}_{\mathrm{x}} \mathrm{MnO}_{3}$ (LSMO) is another kind of Mn-based magnetic materials, which is regarded as one of the most promising spintronics materials for its excellent properties of high Curie temperature and high spin polarizaiton rate
[31]. These properties of perovskite manganese oxide are most interesting for their use in the form of thin film heterostructures for magnetic recording devices, fieldeffect transistors and spin injection devices. However, these electronic and magnetic properties will be influenced by oxygen vacancies which are very common and are not easy to avoid during deposition. To provide deep insights into the roles of oxygen vacancies $[32,33]$, we present a comparative study of the magnetic properties of stoichiometric $\mathrm{La}_{2 / 3} \mathrm{Sr}_{1 / 3} \mathrm{MnO}_{3}$ and nonstoichiometric $\mathrm{La}_{2 / 3} \mathrm{Sr}_{1 / 3} \mathrm{MnO}_{3-\delta}$ by XMCD spectroscopy. The two thin films with the thickness of about $80 \mathrm{~nm}$ [34] were deposited on the $\mathrm{SrTiO}_{3}$ (STO) (001) substrates by molecular beam epitaxy (MBE) under different oxygen pressures.The Curie temperature $\left(T_{c}\right)$ derived from the maximal slope of the $\mathrm{M}(\mathrm{T})$ curve were found to be $350 \mathrm{~K}$ and $165 \mathrm{~K}$ for the stoichiometric and nonstoichiometric films, respectively. Fig. 2 a shows the $\mathrm{Mn}-L_{2,3}$ XAS/XMCD spectra of the $\mathrm{La}_{2 / 3} \mathrm{Sr}_{1 / 3} \mathrm{MnO}_{3} / \mathrm{SrTiO}_{3}$ at an incident angle of $45 \mathrm{deg}$ with a saturation magnetic field of about $0.3 \mathrm{~T}$ at room temperature. Large dichroic signals have been observed for Mn elements from LSMO/STO, indicating that the stoichiometric LSMO is at ferromagnetic phase $[35,36]$ at room temperature. Fig. 2 b shows the comparative results of the Mn XMCD spectra for stoichiometric $\mathrm{La}_{2 / 3} \mathrm{Sr}_{1 / 3} \mathrm{MnO}_{3}$ and nonstoichiometric $\mathrm{La}_{2 / 3} \mathrm{Sr}_{1 / 3} \mathrm{MnO}_{3}-\delta$ samples. 

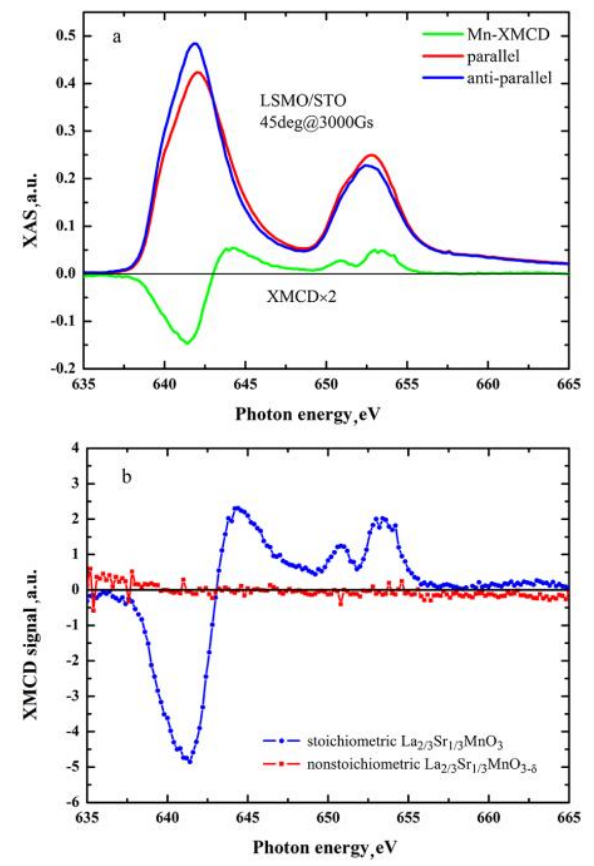

Fig. 2. The XMCD spectra at the $\mathrm{Mn} L_{2,3}$ edge for stoichiometric $\mathrm{La}_{2 / 3} \mathrm{Sr}_{1 / 3} \mathrm{MnO}_{3}$ and nonstoichiometric $\mathrm{La}_{2 / 3} \mathrm{Sr}_{1 / 3} \mathrm{MnO}_{3-\delta}$ samples: a-the Mn- $L_{2,3}$ XAS/XMCD spectrum of the $\mathrm{La}_{2 / 3} \mathrm{Sr}_{1 / 3} \mathrm{MnO}_{3} / \mathrm{SrTiO}_{3}$ at an incident angle of $45 \mathrm{deg}$ with a saturation magnetic field of about $0.3 \mathrm{~T}$ at room temperature; $b$-the XMCD comparative results for both samples

The two samples were measured at the same condition and both dichroic signals were normalized to the sum of the parallel and antiparallel polarized XAS signals. It can be seen that there is no obvious dichroic signal for nonstoichiometric sample, indicating it is at paramagnetic phase at room temperture and the magnetic moment is below the detectable limit of the set-up. Our experimental result clearly demonstrates that the oxygen vacancies degrade the magnetic properties of the LSMO film.

\section{CONCLUSIONS}

Field-reversal and angular dependent XMCD techniques have been established for the 4B7B beamline at BSRF and have been applied to investigate the magnetic properties and magnetic anisotropy of two typical Mnbased materials. The interface ferromagnetism and magnetic anisotropy of the $\mathrm{Fe} /(\mathrm{Ga}, \mathrm{Mn}) \mathrm{As}$ heterostructures have been studied by XAS/XMCD spectroscopy. And enhanced XMCD signals have been found for Fe elements by varying the incidence angle of the bilayer, a relative small but unambiguous magnetic signal were obsevred at Mn $L_{2,3}$ edges even at room temperature, which indicates an antiferromagnetic coupling at the interfaces. A quite large XMCD asymmetry between the surface parallel and normal directions indicates huge magnetocrystalline anisotropy energy of the Fe film in the bilayer. Such interface magnetic coupling and magnetocrystalline anisotropy may provide possible applications for manipulating magnetism via the proximity effect and designing room-temperature DMS devices. We also present a comparative study of the stoichiometric
$\mathrm{La}_{2 / 3} \mathrm{Sr}_{1 / 3} \mathrm{MnO}_{3}$ and nonstoichiometric $\mathrm{La}_{2 / 3} \mathrm{Sr}_{1 / 3} \mathrm{MnO}_{3-\delta} \square$ in order to reveal the role of the oxygen vacancies. The XMCD result clearly demonstrates that the oxygen vacancies degrade the magnetic properties of the perovskite manganese oxide film.

\section{Acknowledgments}

This work is financially supported by the National Natural Science Foundation of China (Grant No. 11505211, 11675206 and 11605225).

\section{REFERENCES}

1. Stöhr, J., Siegmann, H. Magnetism: From Fundamentals to Nanoscale Dynamics. Springer, Berlin, 2006: pp. 389-400. https://doi.org/10.5860/choice.44-4514

2. Thole, B., Carra, P., Sette, F., van der Laan, G. X-Ray Circular Dichroism as a Probe of Orbital Magnetization Physical Review Letters 68 (12) 1992: pp. 1943-1945. https://doi.org/10.1103/PhysRevLett.68.1943

3. Paolo, C., Thole, B., Altarelli, M., Wang, X. X-Ray Circular Dichroism and Local Magnetic Fields Physical Review Letters 70 (5) 1993: pp. 694-697. https://doi.org/10.1103/physrevlett.70.694

4. Radu, F., Abrudan, R., Radu, I., Schmitz, D., Zabel, H. Perpendicular Exchange Bias in Ferrimagnetic Spin Valves Nature Communications 3 2012: $715(1-7)$. https://doi.org/10.1038/ncomms 1728

5. Funk, T., Deb, A., George, S.J., Wang, H., Cramer, S.P. X-Ray Magnetic Circular Dichroism - a High Energy Probe of Magnetic Properties Coordination Chemistry Reviews 249 2005: pp. 3-30. https://doi.org/10.1016/j.ccr.2004.05.017

6. Figueiredo, J.J.S., Basilio, R., Landers, R., Garcia, F., Siervo, A.D. A New Ultra-High-Vacuum Variable Temperature and High-Magnetic-Field X-Ray Magnetic Circular Dichroism Facility at LNLS Journal of Synchrotron Radiation 16 2009: pp. 346-351. https://doi.org/10.1107/s090904950900243x

7. Arenholz, E., Prestemon, S.O. Design and Performance of an Eight-Pole Resistive Magnet for Soft X-Ray Magnetic Dichroism Measurements Review of Scientific Instruments 76 2005: pp. $083908(1-8)$. https://doi.org/10.1063/1.2008027

8. Antonov, V.N., Yaresko, A.N., Jepsen, O. X-Rray Magnetic Dichroism in III-V Diluted Magnetic Semiconductors: First-Principles Calculations Physical Review B 81 2010: pp. 075209 (1-19). https://doi.org/10.1103/physrevb.81.075209

9. Guo, Z.Y., Hong, C.H., Xing, H.Y., Tang, K., Zheng, L., Xui, W., Chen, D.L., Cui, M.Q., Zhao, Y.D. A New Soft X-Ray Magnetic Circular Dichroism Facility at the BSRF Beamline 4B7B Chinese Physics $C \quad 39$ 2015: pp. $048003(1-5)$. https://doi.org/10.1088/1674-1137/39/4/048003

10. Nakamura, T., Suzuki, M. Recent Progress of the X-Ray Magnetic Circular Dichroism Technique for ElementSpecific Magnetic Analysis Journal of the Physical Society of Japan 82 2013: pp. $021006(1-20)$. https://doi.org/10.7566/jpsj.82.021006

11. Muro, T., Nakamura, T., Matsushita, T., Kimura, H., Nakatani, T., Hirono, T., Kudo, T., Kobayashi, K., Saitoh, Y., Takeuchi, M., Hara, T., Shirasawa, K., Kitamura, H. Circular Dichroism Measurement of Soft X- 
Ray Absorption Using Helicity Modulation of Helical Undulator Radiation Journal of Electron Spectroscopy and Related Phenomena 144-147 2005: pp. 1101-1103. https://doi.org/10.1016/j.elspec.2005.01.140

12. Nakagawa, T., Takagi, Y., Matsumoto, Y., Yokoyama, T. Enhancements of Spin and Orbital Magnetic Moments of Submonolayer $\mathrm{Co}$ on $\mathrm{Cu}(001)$ Studied by X-Ray Magnetic Circular Dichroism Using Superconducting Magnet and Liquid He Cryostat Japanese Journal of Applied Physics 47 2008: pp. $2132-2136$.

https://doi.org/10.1143/jjap.47.2132

13. Stöhr, J., König, H. Determination of Spin- and OrbitalMoment Anisotropies in Transition Metals by AngleDependent X-Ray Magnetic Circular Dichroism Physical Review Letters 75 (20) 1995: pp. 3748-3751. https://doi.org/10.1103/physrevlett.75.3748

14. Eelbo, T., Wasniowska, M., Sikora, M., Dobrzanski, M., Kozlowski, A., Pulkin, A., Autes, G., Miotkowski, I., Yazyev, O.V., Wiesendanger, R. Strong Out-of-Plane Magnetic Anisotropy of $\mathrm{Fe}$ Adatoms on $\mathrm{Bi}_{2} \mathrm{Te}_{3}$ Physical Review B 89 2014: pp. 104424 (1-6).

https://doi.org/10.1103/PhysRevB.89.104424

15. Edmonds, K.W., van der Laan, G., Freeman, A.A., Farley, N.R.S., Johal, T.K., Campion, R.P., Foxon, C.T. Gallagher, B.L., Arenholz, E. Angle-Dependent X-Ray Magnetic Circular Dichroism from (Ga,Mn)As: Anisotropy and Identification of Hybridized States Physical Review Letters 96 2006: pp. $117207(1-4)$. https://doi.org/10.1103/physrevlett.96.117207

16. Freeman, A.A. Magnetic X-Ray Spectroscopy Studies of Dilute Magnetic Semiconductors, PhD thesis, University of Nottingham, 2009.

17. Li, R.P., Wang, J., Li, H.H., Guo, Y.X., Wang, F., Hu, Z.W. In-Plane Anisotropy of Iron Single-Crystal Thin Film Using X-Ray Magnetic Circular Dichroism Acta Physica Sinca 54 2005: pp. 3851-3855.

http://dx.chinadoi.cn/10.7498/aps.54.3851

18. Guo, Y.X., Wang, J., Xu, P.S., Li, H.H., Cai, J.W. Element-Specific in-Plane Magnetic Anisotropy in $\mathrm{Co}_{0.9} \mathrm{Fe}_{0.1}$ Films Acta Physica Sinca 56 2007: pp. 1121-1126. http://dx.chinadoi.cn/10.7498/aps.56.1121

19. Dürr, H.A., Guo, G.Y., van der Laan, G., Lee, J., Lauhoff, G., Bland, J.A.C. Element-Specific Magnetic Anisotropy Determined by Transverse Magnetic Circular XRay Dichroism Science 277 1997: pp. 213-215. https://doi.org/10.1126/science.277.5323.213

20. van der Laan, G. Relation Between the Angular Dependence of Magnetic X-Ray Dichroism and Anisotropic Ground-State Moments Physical Review B 57 1998: pp. $5250-5258$.

https://doi.org/10.1103/physrevb.57.5250

21. van der Laan, G., Chopdekar, R.V., Suzuki, Y., Arenholz, E. Strain-Induced Changes in the Electronic Structure of $\mathrm{MnCr}_{2} \mathrm{O}_{4}$ Thin Films Probed by X-Ray Magnetic Circular Dichroism Physical Review Letters 105 2010: pp. $067405(1-4)$. https://doi.org/10.1103/physrevlett.105.067405

22. Mamiya, K., Koide, T., Ishida, Y., Osafune, Y., Fujimori, A., Suzuki, Y., Katayama, T., Yuasa, S. AngleResolved Soft X-Ray Magnetic Circular Dichroism in a Monatomic Fe Layer Facing an $\mathrm{MgO}(001)$ Tunnel Barrier Radiation Physics and Chemistry 75 2006: pp. $1872-1877$. https://doi.org/10.1016/j.radphyschem.2005.07.042

23. Maccherozzi, F., Sperl, M., Panaccione, G., Minar, J., Polesya, S., Ebert, H., Wurstbauer, U., Hochstrasser, M.,
Rossi, G., Woltersdorf, G., Wegscheider, W., Back, C.H. Evidence for a Magnetic Proximity Effect up to Room Temperature at $\mathrm{Fe} /(\mathrm{Ga}, \mathrm{Mn}) \mathrm{As}$ Interfaces Physical Review Letters 101 2008: pp. $267201(1-4)$.

https://doi.org/10.1103/physrevlett.101.267201

24. Olejnik, K., Wadley, P., Haigh, J.A., Edmonds, K.W., Campion, R.P., Rushforth, A.W., Gallagher, B.L., Foxon, C.T., Jungwirth, T., Wunderlich, J., Dhesi, S.S., Cavill, S.A., van der Laan, G., Arenholz, E. Exchange Bias in a Ferromagnetic Semiconductor Induced by a Ferromagnetic Metal: Fe/(Ga,Mn)As Bilayer Films Studied by XMCD Measurements and SQUID Magnetometry Physical Review B 81 2010: pp. 104402 (1-5). https://doi.org/10.1103/physrevb.81.104402

25. Sperl, M., Maccherozzi, F., Borgatti, F., Verna, A., Rossi, G., Soda, M., $\quad$ Schuh, D., $\quad$ Bayreuther, G., Wegscheider, W., Cezar, J.C., Yakhou, F., Brookers, N.B., Back, C.H., Panaccione, G. Identifying the Character of Ferromagnetic $\mathrm{Mn}$ in Epitaxial $\mathrm{Fe} /(\mathrm{Ga}, \mathrm{Mn})$ As Heterostructures Physical Review $B \quad 81$ 2010: pp. $035211(1-6)$.

https://doi.org/10.1103/physrevb.81.035211

26. Alsmadi, A.M., Choi, Y., Keavney, D.J., Eid, K.F., Kirby, B.J., Liu, X., Leiner, J., Tivakornsasithorn, K., Dobrowolska, M., Furdyna, J.K. Interfacial Exchange Coupling in Fe/(Ga,Mn)As Bilayers Physical Review B 89 2014: pp. 224409 (1-7). https://doi.org/10.1103/physrevb.89.224409

27. Sperl, M., Torelli, P., $\quad$ Eigenmann, F., $\quad$ Soda, M., Polesya, S., Utz, M., $\quad$ Bougeard, D., $\quad$ Ebert, H., Panaccione, G., Bank, C.H. Reorientation Transition of the Magnetic Proximity Polarization in Fe/(Ga,Mn)As Bilayers Physical Review B 85 2012: pp. 184428 (1-7). https://doi.org/10.1103/physrevb.85.184428

28. Nie, S.H., Chin, Y.Y., Liu, W.Q., Tung, J.C., Lu, J., Lin, H.J., Guo, G.Y., Meng, K.K., Chen, L., Zhu, L.J., Pan, D., Chen, C.T., Xu, Y.B., Yan, W.S., Zhao, J.H. Ferromagnetic Interfacial Interaction and the Proximity Effect in a $\mathrm{Co}_{2} \mathrm{FeAl} /(\mathrm{Ga}, \mathrm{Mn}) \mathrm{As}$ Bilayer Physical Review Letters 111 2013: pp. $027203(1-5)$. https://doi.org/10.1103/physrevlett.111.027203

29. Song, C., Sperl, M., Utz, M., Ciorga, M., Woltersdorf, G., Schuh, D., Bougeard, D., Back, C.H., Weiss, D. Proximity Induced Enhancement of the Curie Temperature in Hybrid Spin Injection Devices Physical Review Letters 107 2011: pp. $056601(1-4)$. https://doi.org/10.1103/physrevlett.107.056601

30. Dziatkowski, K., Kalbarczyk, K., Szczytko, J., Gosk, J., Tokarczyk, M., $\quad$ Kowalski, G., $\quad$ Twardowski, A., Bednarski, W., Ostrowski, A., Waplak, S., Martinek, J., Liu, X., Furdyna, J.K. Interplay of Magnetic Anisotropies in Epitaxial Ferromagnetic Hybirds of $\mathrm{Fe}$ and $(\mathrm{Ga}, \mathrm{Mn}) \mathrm{As}$ Journal of the Magnetics Society of Japan 38 2014: pp. $111-1$. https://doi.org/10.3379/msjmag.1402r012

31. Wang, K., Tang, M.H., Xiong, Y., Li, G., Xiao, Y.G., Zhang, W.L., Wang, Z.P., Li, Z., He, J. Epitaxial Growth and Magnetic/Transport Properties of $\mathrm{La}_{0.7} \mathrm{Sr}_{0.3} \mathrm{MnO}_{3}$ Thin Films Grown on $\mathrm{SrTiO}_{3}$ with Optimized Growth Conditions RSC Advances 7 2017: pp. 31327-31332. https://doi.org/10.1039/c7ra04356b

32. Alharbi, A.A., Alkahtani, M., Al-Dossary, O. The Role of Oxygen Vacancies on Magnetic Properties of LSMO AIP Conference Proceedings 1370 2011: pp. 116. https://doi.org/10.1063/1.3638091 
33. Feng, Y.Q., Jin, K.J., Gu, L., He, X., Ge, C., Zhang, Q.H., He, M., Guo, Q.L., Wan, Q., He, M., Lu, H.B., Yang, G.Z Insulating Phase at Low Temperature in Ultrathin La $0.8 \mathrm{Sr}_{0.2} \mathrm{MnO}_{3}$ films Scientific Reports 6 2016: pp. 22382. https://doi.org/10.1038/srep22382

34. Shibata, G., Yoshimatsu, K., Sakai, E., Singh, V.R., Verma, V.K., Ishigami, K., Harano, T., Kadono, T., Takeda, Y., Okane, T., Saitoh, Y., Yamagami, H., Sawa, A., Kumigashira, H., Oshima, M., Koide, T., Fujimori, A. Thickness-Dependent Ferromagnetic Metal to Paramagnetic Insulator Transition in $\mathrm{La}_{0.6} \mathrm{Sr}_{0.4 \mathrm{MnO}}$ Thin Films Studied by X-Ray Magnetic Circular Dichroism Physical Review B 89 2014: pp. 235123. https://doi.org/10.1103/physrevb.89.235123
35. Lee, J.S., Arena, D.A., Yu, P., Nelson, C.S., Fan, R., Kinane, C.J., Langridge, S., Rossell, M.D., Ramesh, R., Kao, C.C. Hidden Magnetic Configuration in Epitaxial La1${ }_{x} \mathrm{Sr}_{x} \mathrm{MnO}_{3}$ Films Physical Review Letters 105 2010: pp. 257204. https://doi.org/10.1103/physrevlett.105.257204

36. Kavich, J.J., Warusawithana, M.P., Freeland, J.W., Ryan, P., Zhai, X., Kodama, R.H., $\quad$ Eckstein, J.N. Nanoscale Suppression of Magnetization at Atomically Assembled Manganite Interfaces: XMCD and XRMS Measurements Physical Review B 76 2007: pp. 014410. https://doi.org/10.1103/physrevb.76.014410 